\title{
Malnutrition nach biliopankreatischer Teilung - postoperative Maßnahmen und Komplikationen
}

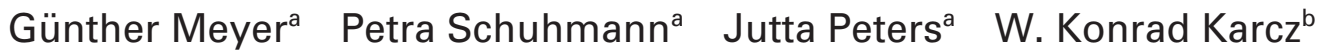 \\ ${ }^{a}$ AMC-Wolfartklinik, Zentrum für Adipositas- und Metabolische Chirurgie, München-Gräfelfing, \\ ${ }^{\mathrm{b}}$ Abteilung Allgemein- und Viszeralchirurgie mit Poliklinik, Universitätsklinikum Freiburg, Deutschland
}

\section{Schlüsselwörter \\ Biliopankreatische Teilung · Adipositaschirurgie · Malnutrition - Mangelerscheinungen · Komplikationen}

\section{Zusammenfassung}

Die biliopankreatische Teilung stellt derzeit die Maximalvariante der bariatrischen Chirurgie dar. Sie weist eine Reihe charakteristischer chirurgischer Komplikationsmöglichkeiten neben den in der bariatrischen Chirurgie generell vorhandenen Risiken auf. Hinzu kommt das dem Verfahren immanente Risiko der Entwicklung von Mangelzuständen im Langzeitverlauf zu einem relevanten Prozentsatz. Diese Mangelzustände können durch eine konsequente Supplementation im Rahmen eines strukturierten Nachsorgeprogramms weitgehend vermieden werden und sind durch eine Substitutionstherapie wieder zu beheben. Möglicherweise kann das Risiko der Malnutrition in der Zukunft durch technische Modifikationen des Verfahrens bei gleich großer bariatrischer Effektivität gesenkt werden. Bei sehr guten Langzeitergebnissen in Bezug auf eine stabile Gewichtsreduktion in Verbindung mit einer hohen Rückbildungsrate der Komorbiditäten erscheint das Malnutritionsproblem bei konsequentem postoperativen Management von untergeordneter Bedeutung. Voraussetzung ist aber eine dementsprechende Compliance des Patienten.

\section{Einleitung}

Die biliopankreatische Teilung (BPD) als derzeit invasivstes, aber auch vermutlich effektivstes Verfahren der bariatrischen Chirurgie kommt vor allem bei extremer Adipositas und/oder

\section{Keywords}

Biliopancreatic diversion - Bariatric surgery · Malnutrition · Nutritional deficiencies · Complications

\section{Summary \\ Malnutrition after Biliopancreatic Diversion - Postoperative Management and Complications}

The biliopancreatic diversion is currently the maximum variant of bariatric surgery. It presents several opportunities of typical surgical complications, beside the risks generally prevailing in bariatric surgery. In addition, there is the procedure-immanent risk of development of nutritional deficiencies in the long-term process, at significant percentages. These deficiencies can, to a great extent, be avoided by consistent supplementation within the scope of a structured follow-up program; they can also be corrected by a substitution therapy. In the future, the risk of malnutrition can possibly be lowered by technically modified procedures with equal bariatric effectiveness. Considering the very good long-term results with regard to stable weight reduction in connection with a high recovery rate of the comorbidities, combined with a consistent postal-surgical management, the problem of malnutrition appears to be of minor importance. However, an appropriate compliance of the patient is also required.

\section{KARGER \\ Fax +497614520714 \\ Information@Karger.de}

www.karger.com (c) 2011 S. Karger GmbH, Freibur

$1662-4025 / 11 / 0047-0034 \$ 38.00 / 0$

Accessible online at:

www.karger.com/ofa
Komorbiditäten des Stoffwechsels - insbesondere dem Diabtes mellitus Typ 2 - zur Anwendung. Dabei gibt es verschiedene Modifikationen mit eigenen Vor- und Nachteilen, die es bei der Indikationsstellung zu berücksichtigen gilt. Die derzeit verbreitesten Varianten sind die BPD-Duodenalswitch (DS) 
Abb. 1. Verfahren der BPD. Aus: Weiner R: Adipositaschirurgie, 1. Aufl., 2009. (C) Elsevier $\mathrm{GmbH}$, Urban \& Fischer, München.
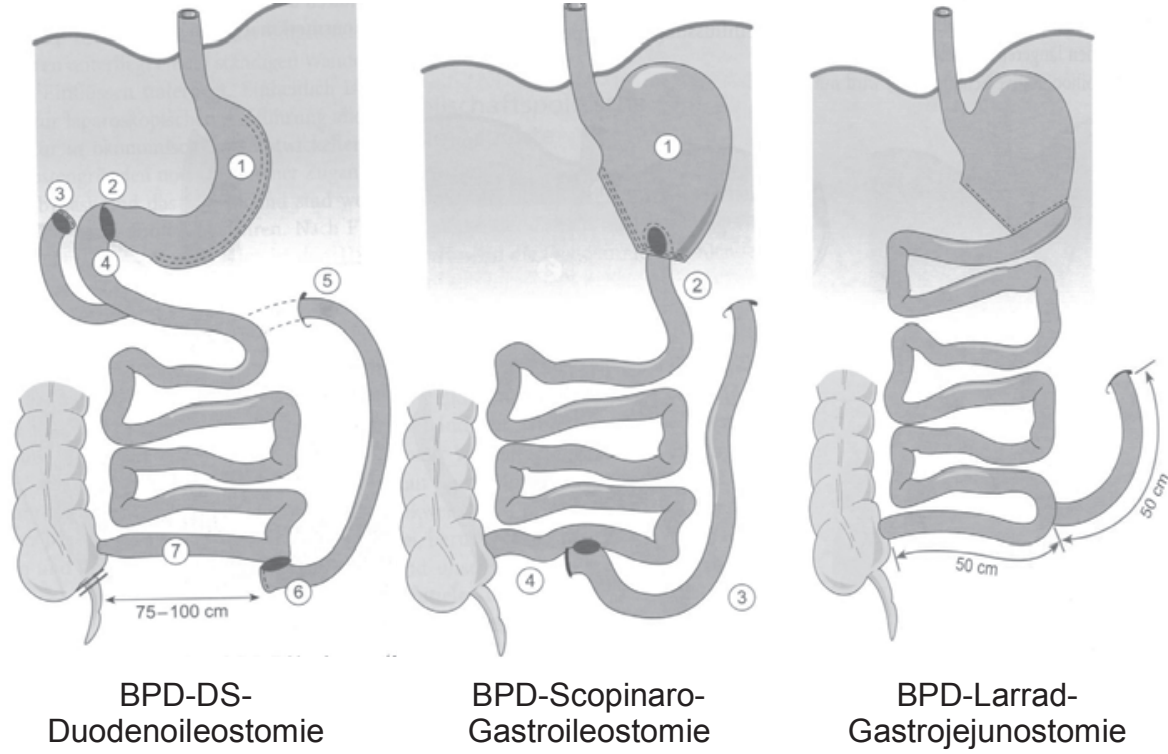

BPD-LarradGastrojejunostomie
[1], die ursprüngliche BPD-Scopinaro [2] und die BPD-Larrad [3]. Alle Verfahren können vollständig laparoskopisch durchgeführt werden.

Prinzip der BPD ist die Kombination einer Magenverkleinerung mit einer langstreckigen Teilung des Dünndarms in einen alimentären Schenkel zum Nahrungstransport sowie einen biliopankreatischen Schenkel zum Transport der Verdauungssäfte. Beide münden sehr tief in einen je nach Verfahren 50-100 cm langen gemeinsamen Kanal vor der Bauhin'schen Klappe (Abb. 1). Bei grundsätzlich definierter Länge des gemeinsamen Kanals haben bei der BPD-DS und BPD-Scopinaro der alimentäre Schenkel und bei der BPDLarrad der biliopankreatische Schenkel exakt abgemessene Längen. Bei Letzterer ist der alimentäre Schenkel am längsten angelegt, wodurch Mangelerscheinungen und insbesondere Hypoproteinämien verringert werden sollen. Die große bariatrische Langzeitwirksamkeit der BPD erklärt sich überwiegend aus einer Fettmangelverwertung, deren Ausmaß von der Länge des gemeinsamen Kanals abhängig ist. Dabei ist die zusätzliche restriktive Wirkung des beim DS angelegten Schlauchmagens stärker als die des bei den anderen Verfahren für die Nahrungspassage belassenen Magenrestes mit einem Fassungsvermögen von 200-300 ml.

Die Methode wirkt somit überwiegend über eine Kombination von Maldigestion und Malabsorption durch die Magenteilresektion, verspäteten Kontakt des Nahrungsbreis mit Gallensäuren und Pankreasenzymen bzw. Verkürzung des zur Resorption zur Verfügung stehenden Dünndarmabschnittes. Im englischen Sprachgebrauch wird keine Unterscheidung zwischen Maldigestion und Malabsorption gemacht, sondern für beide der Begriff «Malabsorption» verwendet. Daher sprechen wir generell von «malabsorptiven» Verfahren. Der Grad der Malabsorption insbesondere für Fett ist dabei bei der BPD-Scopinaro und -Larrad mit einem exakt nur $50 \mathrm{~cm}$ langen gemeinsamen Kanal noch stärker als bei der BPD-DS ausgeprägt [4],

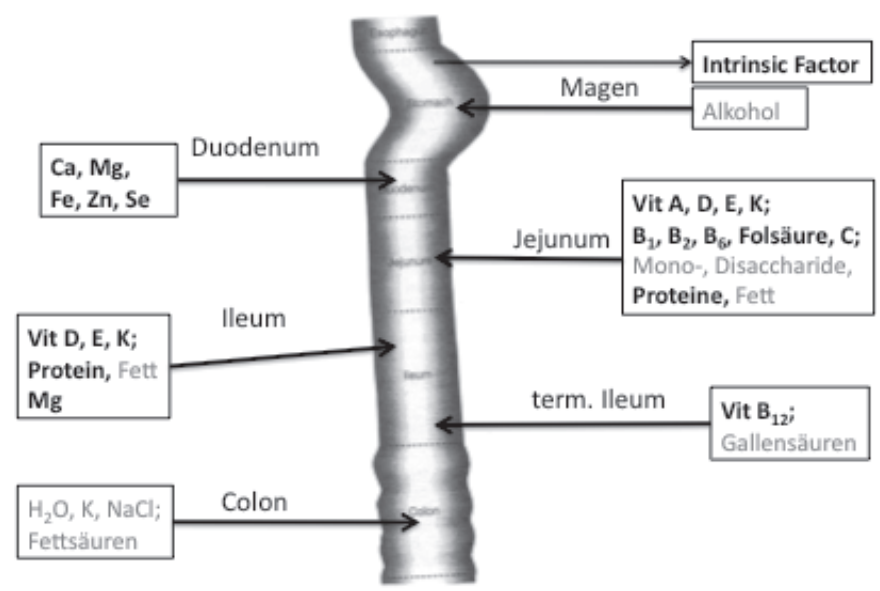

Abb. 2. Nährstoffresorption im Gastrointestinaltrakt.

deren gemeinsamer Kanal $75-100 \mathrm{~cm}$ beträgt. Andererseits wird bei der sogenannten Larrad-50-50-BPD im Gegensatz zu den beiden anderen Varianten nur ein kleinerer Teil des Jejunums von der Nahrungspassage ausgeschaltet, wodurch die Malabsorption insgesamt verringert werden soll (Abb. 2). Leitsymptome sind eine Gewichtsabnahme, die die bariatrische Chirurgie therapeutisch nutzt, verfahrens- und diätabhängige Fettstühle, Flatulenz und Diarrhö sowie mögliche Mangelsymptome.

\section{Betroffene Substanzen}

Die Gefahr der Entwicklung von Mangelerscheinungen insbesondere im Langzeitverlauf ist bei der BPD deutlich größer als bei den anderen bariatrischen Verfahren. Sie ist Folge der Malnutrition durch Malabsorption bei mangelhafter Verdauung und Resorption und droht insbesondere bei ungenügender postbariatrischer Supplementation, fal- 
Tab. 1. Standard-Supplementation der AMCWolfartklinik, modifiziert nach $[9,10]$

\begin{tabular}{ll} 
MVP mit Spurenelementen & $200 \%$ des Tagesbedarfes \\
Vitamin B12 & $1000 \mu \mathrm{g} / 3$ Monate (intramuskulär) \\
Folsäure (in MVP) & $400 \mu \mathrm{g} / \mathrm{Tag}$ \\
Calcium (inkl. Calcium aus der Nahrung) & $2000 \mathrm{mg} / \mathrm{Tag}, 3-4 \times 500 \mathrm{mg}$ \\
Eisen (teilweise in MVP) & $50-100 \mathrm{mg} / \mathrm{Tag}$ (bei prämenopausalen Frauen) \\
& sonst etwa $18 \mathrm{mg} / \mathrm{Tag}$ \\
Vitamin D3 & $1500-2000 \mathrm{IE} / \mathrm{Tag}$ \\
Vitamin A (in MVP) & $10000 \mathrm{IE} / \mathrm{Tag}$ \\
Vitamin E (in MVP) & $22 \mathrm{mg} / \mathrm{Tag}$ \\
Vitamin K (eventuell in MVP) & $300 \mu \mathrm{g} / \mathrm{Tag}$ \\
Zink (in MVP) & $15 \mathrm{mg} / \mathrm{Tag}$ \\
Eiweiß & $80-90 \mathrm{~g} / \mathrm{Tag}$ \\
\hline
\end{tabular}

MVP = Multivitaminpräparat.
Tab. 2. Häufigkeiten von Mängeln bei BPD-DS und BPD-Scopinaro $[9,11-17]$

\begin{tabular}{ll}
\hline Mangelerscheinung & $\%$ \\
\hline Hypoproteinämie & $1,1-21$ \\
Vitamin-A-Mangel & $61-69$ \\
Nachtblindheit & bis 8 \\
Vitamin-D-Mangel & 63 \\
Hypokalzämie & $25-48$ \\
Sekundärer Hyperparathyreoidismus & $69-90$ \\
Vitamin-E-Mangel & 4 \\
Vitamin-K-Mangel (keine klinische & 68 \\
Manifestation) & \\
Vitamin-B12-Mangel & $12-22$ \\
Eisenmangel & 44 \\
Selenmangel & 15 \\
Zinkmangel & 51 \\
Magnesiummangel & 5 \\
\hline
\end{tabular}

scher Ernährung und fehlender Nachsorge. Dabei sind wegen der Magenteilresektion und der Ausschaltung des Duodenums sowie unterschiedlich großen Teilen des Jejunums von der Nahrungspassage vor allem die fett- und wasserlöslichen Vitamine, Mineralstoffe, Spurenelemente und Eiweiße betroffen (Abb. 2). Die Mangelerscheinungen werden klinisch relevant, wenn keine ausreichende Supplementation und Kontrolle erfolgt [5-8].

\section{Postoperative Maßnahmen}

Wesentliche Maßnahmen zur Prävention einer klinisch relevanten Malnutrition sind eine prinzipielle und individuelle Supplementation sowie bilanzierte Ernährung und regelmäßige Nachsorge mit laborchemischen Kontrollen. Jährlich sollten dabei auch Knochendichtemessungen und Gebisskontrollen erfolgen. Basis der täglichen Supplementation ist die Einnahme eines Multivitaminpräparates mit Spurenelementen zu 200\% des Tagesbedarfes. In der Regel benötigen die Patienten eine darüber hinausgehende Ergänzung (Tab. 1).
Mangelerscheinungen betreffen besonders häufig das Vitamin D und Calcium, mit Entwicklung eines sekundären Hyperparathyreoidismus, sowie Eisen und Zink. Ebenfalls häufig auftretende Vitamin-A- und -K-Mängel müssen beachtet werden, führen aber nur selten zu klinischen Problemen (Tab. 2). Abhängig vom individuellen Befund müssen erniedrigte Substanzen zusätzlich supplementiert werden. Auf ausreichende Eiweißzufuhr ist zu achten. Vitamin B12 muss regelmäßig verabreicht werden.

Möglicherweise können Komplikationen der Malnutrition durch die von Larrad entwickelte Modifikation verringert werden. In einem 10-Jahres-Nachbeobachtungszeitraum fand er bei gutem bariatrischen Ergebnis nur in 0,29\% eine schwere Hypoproteinämie, in $45 \%$ einen in $28 \%$ bereits präoperativ vorhanden gewesenen sekundären Hyperparathyreoidismus und in 30\% einen Eisenmangel. Die Revisionsoperationsrate lag bei $0 \%$ [18].

\section{Malnutritionsbedingte Komplikationen}

Im Rahmen der Nachsorgeuntersuchungen sollen sich anbahnende oder bereits vorhandene Mängel durch laborchemische und klinische Kontrollen erfasst und mittels entsprechender Substitution therapiert werden. Generelle Symptome einer Malnutrition sind Müdigkeit, Schwindel und Zeichen einer verminderten Immunantwort. Spezifische Symptome weisen auf bestimmte Mangelzustände hin. Die Erstellung eines Ernährungsprotokolls kann zur ätiologischen Abklärung und Therapiefestlegung hilfreich sein. Unbehandelt drohen geistige und/ oder körperliche Schwäche, Krankheit und letztlich der Tod.

\section{Protein}

Ein Eiweißmangel ist abhängig vom Magenvolumen, der Länge des alimentären Schenkels, der individuellen Resorption und den Essgewohnheiten des Patienten [9]. Er kann zu Schwäche, Anämie, Ödembildung und Haarverlust führen [17]. Eine orale, enterale oder parenterale Substitution erfolgt mit bis zu 2 g/kg (Idealgewicht) Protein pro Tag. 


\section{Vitamine}

Auf die fettlöslichen Vitamine muss bei eingeschränkter Fettresorption besonders geachtet werden. Vitamin-A-Mangel kann zu Nachtblindheit führen [17] und wird mit einer Substitution von 50000 IE/2 Wochen bis 10000 IE/Tag behandelt. Vitamin-D-Mangel führt zu sekundärem Hyperparathyreoidismus. Die Substitution kann bis zu 50000 IE/Woche über 8 Wochen in Kombination mit Calciumgaben gesteigert werden und sollte getrennt von Eisengaben erfolgen. Bei Feststellung einer Osteoporose wird diese entsprechend therapiert, beispielsweise mit Bisphosphonaten [4]. Vitamin-E-Mangel tritt sehr selten auf [15] und kann muskuläre sowie Zentralnervensystem (ZNS)-Symptome hervorrufen. Er wird mit bis zu $500 \mathrm{mg} /$ Tag substituiert. Blutungen bei Vitamin-K-Mangel sind eine Rarität [15]. Die Substitution erfolgt mit $300 \mu \mathrm{g} / \mathrm{Tag}$.

Ein symptomatischer Vitamin-B1-Mangel ist selten und kann zur Beriberi-Krankheit sowie Wernicke-Encephalopathie führen. Die Therapie besteht in Thiamin-Infusionen mit $50-150 \mathrm{mg} / \mathrm{Tag}$. Ebenfalls selten kommt es zu Exanthemen und Hautrissen bei Vitamin-B2-Mangel sowie Dermatitis, Glossitis, Cheilosis oder Anämie infolge eines VitaminB6-Mangels. Die Therapie erfolgt üblicherweise durch die $200 \%$ ige Multivitamin-Supplementation. Folsäuremangel kann zur megaloblastären Anämie führen und wird mit einer Substitution von $1000 \mu \mathrm{g} / \mathrm{Tag}$ behandelt. Eine megaloblastäre Anämie - selten eine funikuläre Spinalerkrankung - kann auch Folge eines Vitamin-B12-Mangels sein. Die Therapie besteht in der Gabe von $1000 \mu \mathrm{g} /$ Monat.

Ein Vitamin-C-Mangel tritt selten auf und führt zu Symptomen wie Leistungsschwäche, Immundefizit, schlechter Wundheilung bis hin zum Skorbut. Die Behandlung erfolgt mit der 200\%igen Multivitamin-Supplementation. Ein Biotinmangel kann einen Haarverlust sowie weitere Hautstörungen bedingen und wird mit $30 \mathrm{mg}$ (+ Protein, Zink)/Tag behandelt.

\section{Mineralstoffe}

Ein Mineralstoffmangel betrifft vor allem das Calcium und selten Magnesium. Bei Osteoporose wird mit $4 \times 500 \mathrm{mg}$ Ca-Citrat oder Ca-Carbonat/Tag substituiert, wobei auf eine von Eisengaben getrennte Zufuhr geachtet werden sollte. Postoperative Magnesiummangelerscheinungen sind äußerst selten [6, 17]. Bei Muskelschwäche oder Muskelkrämpfen, Apathie oder Arrhythmien wird Magnesium mit 450 mg/Tag gegeben.

\section{Spurenelemente}

Ein Mangel an Spurenelementen wird im Verlauf nicht selten beobachtet. Dies betrifft insbesondere das Eisen und eine hierdurch bedingte Anämie. Die notwendige Substitution insbesondere nach Verfahren mit Duodenalexklusion erfolgt optimalerweise in Kombination mit Vitamin C oral [9], macht aber häufig bei schlechter Resorption eine parenterale Verabreichung nötig [19]. Sie sollte zeitlich getrennt von VitaminD3- und Calcium-Substitution erfolgen. Da die Zinkresorption von der Fettresorption abhängig ist, werden Defizite häufig beobachtet $[9,15,17]$. Zinkmangel kann zu Haarausfall, Diarrhö und neuropsychologischen Störungen führen und wird mit bis zu $30 \mathrm{mg} / \mathrm{Tag}$ therapiert. Selenmangel ist selten [6, 14, 17] und kann Ursache einer Kardiomyopathie (Keshan-Krankheit) sein. Die Substitutionstherapie besteht in bis zu $300 \mu \mathrm{g} /$ Tag. Ein klinisch relevanter postoperativer Kupfermangel stellt eine absolute Rarität dar, der dann zu einer Anämie sowie Myelopathie führen kann. Die Substitution erfolgt mit bis zu $2 \mathrm{mg} / \mathrm{Tag}$ [20].

Die Therapiemöglichkeiten der Malnutrition bei der BPD erstrecken sich somit von der Ernährungsschulung und -therapie (Ernährungsprotokoll, Nährstoffberechnung) über die Supplementation von Protein, Vitaminen und Mineralstoffen sowie Spurenelementen bis zur enteralen oder parenteralen Substitutionstherapie. Nur in sehr seltenen Ausnahmen wird bei Versagen dieser Maßnahmen und gravierender Symptomatik eine Redo-Operation mit Umwandlung notwendig.

Wenngleich die Malnutrition ein verfahrensimmanentes Problem der BPD ist, sind die Mangelerscheinungen selten schwerwiegend und können bei konsequenter Supplementation, bilanzierter Ernährung sowie regelmäßigem Follow-up in aller Regel durch entsprechende Substitution erfolgreich behandelt werden.

\section{Disclosure Statement}

Es bestehen keine Interessenkonflikte.

\section{Literatur}

1 Hess DS, Hess DW: Biliopancreatic diversion with a duodenal switch. Obes Surg 1998;8:267-282.

2 Scopinaro N, Gianetta E, Civalleri D, Bonalumi U, Bachi V: Biliopancreatic bypass for obesity: II. Initial experience in man. Br J Surg 1979;66:613-620.

3 Larrad-Jiménez A, Sánchez Cabezudo C, de Quadros Borrajo PP, Garcia R, Esteban BM, Garcia Robles R: Course of metabolic syndrome following the biliopancreatic diversion of Larrad. Obes Surg 2004;14:1176-1181.
4 Raab H, Weiner R: Ernährung, Mangelerscheinungen und Supplemente; in Weiner RA (Hrsg): Adipositaschirurgie. München, Elsevier, 2010, pp 291-318.

5 Alvarez-Leite JI: Nutrient deficiencies secondary to bariatric surgery. Curr Opin Clin Nutr Metab Care 2004;7:569-575.

6 Bloomberg RD, Fleishman A, Nalle JE, Herron DM, Kini S: Nutritional deficiencies following bariatric surgery: what have we learned? Obes Surg 2005;15:145-154.
7 Ludwig K, Schneider-Koriath S, Prinz C, Bernhardt J: Nachsorge und Supplementation nach Adipositaschirurgie. Adipositas 2008;2:26-30.

8 Malone M: Recommended supplements for bariatric surgery patients. Ann Pharmacother 2008;42: 1851-1858.

9 Aills L, Blankenship J, Buffington C, Furtado M, Parrott J: ASMBS allied health nutritional guidelines for the surgical weight loss patient. Surg Obes Relat Dis 2008;4:S73-S108. 
10 AACE/TOS/ASMBS Guidelines - American Association of Clinical Endocrinologists, The Obesity Society, and American Society for Metabolic and Bariatric Surgery: Medical guidelines for clinical practise for the perioperative nutritional, metabolic, and non-surgical support of the bariatric surgery patient. Surg Obes Relat Dis 2008;4:S109-S184.

11 Totté E, Hendrickx L, van Hee R: Biliopancreatic diversion for treatment of morbid obesity: experience in 180 consecutive cases. Obes Surg 1999;9: 161-165.

12 Skroubis G, Sakellaropoulos G, Pouggouras K Mead N, Nikiforidis G, Kalfarentzos F: Comparison of nutritional deficiencies after Roux-en-Y gastric bypass and after biliopancreatic diversion with Roux-en-Y gastric bypass. Obes Surg 2002;12:551558.
13 Hamoui N, Kim K, Anthone G, Crookes PF: The significance of elevated levels of parathyroid hormone in patients with morbid obesity before and after bariatric surgery. Arch Surg 2003;138:891-897.

14 Dolan K, Hatzifotis M, Newbury L, Lowe N, Fielding G: A clinical and nutritional comparison of biliopancreatic diversion with and without duodenal switch. Ann Surg 2004;240:51-56.

15 Slater GH, Ren CJ, Siegel N, Williams T, Barr D, Wolfe B, Dolan K, Fielding GA: Serum fat-soluble vitamin deficiency and abnormal calcium metabolism after malabsorptive bariatric surgery. J Gastrointest Surg 2004;8:48-55.

16 Ocón Bretón J, Perez Naranjo S, Gimeno Laborda S, Benito Ruesca P, Garcia Hernández R: Effectiveness and complications of bariatric surgery in the treatment of morbid obesity. Nutr Hosp 2005; 20:409-414.
17 Davies DJ, Baxter JM, Baxter JN: Nutritional deficiencies after bariatric surgery. Obes Surg 2007;17: 1150-1158

18 Larrad-Jiménez A, Diaz-Guerra CS, de Cuadros Borrajo P, Lesmes IB, Esteban BM: Short-, midand long-term results of Larrad biliopancreatic diversion. Obes Surg 2007;17:202-210.

19 Varma S, Baz W, Badine E, Nakhl F, McMullen H, Nicastro J, Forte F, Terjanian T, Dai Q: Need for parenteral iron therapy after bariatric surgery. Surg Obes Relat Dis 2008;4:715-719.

20 DACH: Referenzwerte für die Nährstoffzufuhr/ Deutsche Gesellschaft für Ernährung (DGE), 1. Aufl. Frankfurt, Umschau/Braus, 2000. 